\title{
A Tie-line Power Smoothing Method for a building Microgrid with Plug-in Electric Vehicles
}

\author{
Kai Yuan ${ }^{1 *}$, Chongbo Sun ${ }^{1}$, Yi Song ${ }^{1}$, and Zhili $\mathrm{Wu}^{1}$ \\ ${ }^{1}$ State Grid Economic and Technological Research Institute Co. Ltd., Beijing, China
}

\begin{abstract}
Aiming to facilitate a high penetration of renewable generation and the low energy consumption technologies at the demand side, there is significant development of low-carbon building Microgrids integrated with renewable generation. However, renewable generation is usually intermittent, uncertain and uncontrollable, which induces power mismatches between power demand and supply for the low-carbon building Microgrids. Therefore, a two-layer method for a building Microgrid is proposed to smooth the tieline power by scheduling the thermal mass of the building and the electric vehicles at two different time scales. A Vehicle-to-Building control strategy was developed to dispatch the electric vehicles as a flexible resource. Numerical studies demonstrated that the proposed method can smooth the fluctuations of the tie-line power for the building Microgrid.
\end{abstract}

\section{Introduction}

Increasing attention is being paid to technologies in renewable energy and energy efficiency improvement due to the rapid growth of global energy use and environmental deterioration 1. Aiming to facilitate a high penetration of renewable generation at the demand side, there is significant development of low-carbon buildings integrated with renewable generation 2. The Microgrid provides an opportunity for the collaborative optimization and management of various energy systems in the buildings 3 .

The existing research work has made good contributions to the energy management for a building Microgrid. The operational performance of a Microgrid in a building in Hong Kong was studied considering operating cost and environmental constraints 2 . A multiobjective dispatch model was proposed in 4 to minimize the daily operating cost and the pollutants emission. An electric chiller (EC) was used as the cooling system of a building in 5 and the electric power consumption of the EC was dispatched using a nonlinear programming method for cost saving.

However, the existing research work mainly focuses on reducing operating cost of the building Microgrid. The renewable generation is usually intermittent, uncertain and uncontrollable, which induces power mismatches between power demand and supply for the building Microgrid. The fluctuations of tie-line power of the building Microgrid are also induced by the mismatches. Therefore, both operating cost reduction and smoothing fluctuations of tie-line power should be considered in the building Microgrid energy management.

Moreover, the flexibility of the building with heat inertia and the flexibility of the plug-in EVs in the building Microgrid haven't been fully explored in the tieline power smoothing for the building Microgrid. Furthermore, due to the different response characteristics of the building thermal mass and the EVs, the two flexible resources (i.e., the building thermal mass and the EVs) should be managed and coordinated at different time scales to make the energy management more efficient.

Therefore, a two-layer method for a building Microgrid is proposed to smooth the tie-line power by scheduling the thermal mass of the building and the electric vehicles at two different time scales. A building is simplified to a lumped thermal mass and modelled as a simplified thermal storage system, namely the virtual energy storage system (VESS). The VESS is dispatched as a flexible resource to smooth the fluctuations of tie-line power of the building Microgrid. A Vehicle-to-Building control strategy is developed to dispatch the electric vehicles as another flexible resource. The VESS and the EVs under V2B control are dispatched at two different time scales considering their different response characteristics, which makes the method more efficient. Numerical studies demonstrated that the proposed method can smooth the fluctuations of the tie-line power for the building Microgrid.

\section{Model of the building microgrid}

\subsection{Configuration of the building microgrid}

A building Microgrid is adopted as the test system in this paper, as shown in Fig. 1. The building Microgrid includes a photovoltaic system, an EC and a V2B system (The EVs under V2B control is abbreviated as V2B system in this paper). In the Microgrid, the electricity

* Corresponding author: yuankai83@126.com 
suppliers are the photovoltaic system and the external power grid. And the electricity consumers are the EC, the lighting and office appliances. Meanwhile, there is a balance between the cooling supply and demand. The building is the cooling consumer and the EC is the cooling supplier.

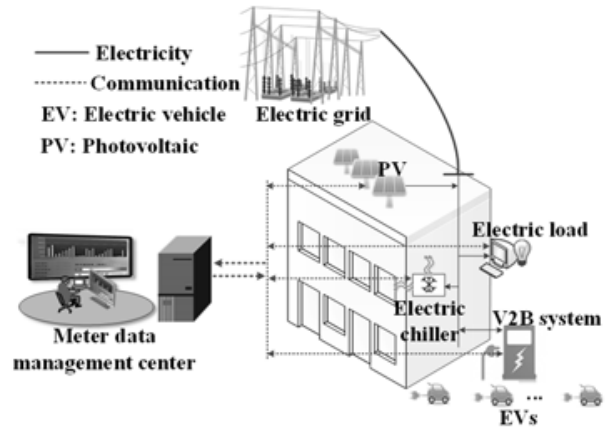

Fig. 1. Configuration of the building Microgrid.

\subsection{Configuration of the building microgrid}

Considering a summer cooling scenario, a building is modeled as a single isothermal air volume 6 . Then, the model of VESS is developed considering the thermal performance of the building. The basic idea of the VESS is that the cooling demand of the building can be adjusted in the energy management process without disturbing the temperature comfort level due to the thermal mass of the building. Therefore, the cooling energy generated by the $\mathrm{EC}$ is stored in the building when the electricity price is low, i.e., the EC is started in advance or the power consumption of the EC is increased. In that case, the VESS is charged in the view point of the Microgrid. In the same way, the cooling energy generated by the EC is discharged in the building when the electricity price is high, i.e., the EC is shut down in advance or the power consumption of the EC is decreased. In that case, the VESS is discharged in the view point of the Microgrid. The indoor temperature comfort zone and temperature set-point are considered in the model of VESS to maintain the customer comfort level. The specific model of VESS can be found in our previous study 78 .

\subsection{The Vehicle-to-Building (V2B) model}

The V2B model consists of three models: 1) the EV battery model; 2) the model of EV mobility behaviour; and 3) the operation constraints of the EV. The generic EV battery model developed in 9 is used to obtain the relationship between the SOC and the charging/discharging behaviours of the EVs.

- EV battery model

Since this paper focuses on the EVs in a building, the type of the EVs is determined as M1 with Home Based Work (HBW) transportation pattern according to a depth survey of the worldwide EV battery market 10 . According to 10 , the probability density function ( $p d f)$ of EV battery capacity (Cap) of M1 EV is defined as the truncated Gamma distribution, as shown in Eq. (1).

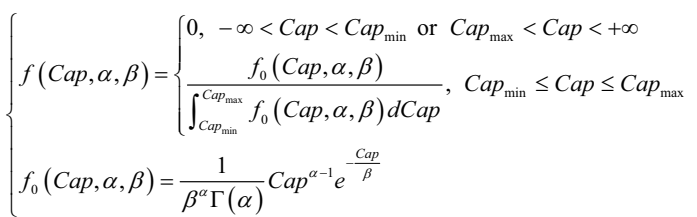

$\alpha$ and $\beta$ are the shape parameter and the scale parameter of the $p d f$ of Gamma distribution; $C_{a p}$ min and $C_{a p_{\max }}$ are the minimum and maximum battery capacities of M1 EV. For M1 EV, the $\alpha$ is 4.5 , the $\beta$ is 6.3 , the $\operatorname{Cap}_{\text {min }}$ is $10 \mathrm{~kW}$ and the Cap $_{\max }$ is $72 \mathrm{~kW}$.

- Model of EV mobility behaviour

It is assumed that all the EVs investigated in this paper are charged at home. They are charged to their expected SOCs $\left(S O C_{d, \text { home }}\right)$ before travelling to satisfy their energy demands travelling from home to work. The $S O C_{d}$, home varies uniformly in the range of $[80 \%, 90 \%]$ to satisfy the travelling demands of the EV users and maintain the life time of a battery from overcharge 9 . It is assumed that SOC drops linearly with the travel distance. The SOC of an EV when it arrives at the $\left(S O C_{i n}\right)$ is calculated by Eq. (2). Since the EVs are charged to $S O C_{d}$, home at home, the minimum expected SOC at the plug-out time when they leave the office $\left(S O C_{d}\right.$, office $)$ is set to be the same as the $S O C_{i n}$.

$$
S O C_{i n}=S O C_{d, h o m e}-\frac{D_{h-w} \times C_{\mathrm{e}}}{C a p}
$$

The distribution of M1 EV of the $C_{e}$ can be found in $10 ; D_{h-w}$ is assumed to be half of the EV's daily travelling distance $(D)$ according to HBW EVs' mobility behavior 10 .

$D$ basically follows a Normal distribution, as shown in Eq. (3) 11.

$$
f\left(D, \mu_{d}, \sigma_{d}\right)=\frac{1}{\sigma_{d} \sqrt{2 \pi}} e^{-\left(D-\mu_{d}\right)^{2} / 2 \sigma_{d}^{2}}
$$

$\mu_{d}$ is the mean value of daily travelling distance; $\sigma_{d}$ is the standard deviation. For HBW vehicles, the $\mu_{d}$ is 35.9 $\mathrm{km}$ and the $\sigma_{d}$ is $19.6 \mathrm{~km} 12$.

The plug-in time $\left(t_{i n}\right)$ when the EVs arrive at the office and the plug-out time $\left(t_{\text {out }}\right)$ when the EVs leave the office are determined based on the office time, which are assumed to follow the Normal distribution 13. Based on the information supplied by the battery characteristics and mobility behaviors, the Monte Carlo simulation method is used to generate the $\mathrm{Cap}, S O C_{i n}, t_{\text {in }}$ and $t_{\text {out }}$ for all the EVs.

- Operation constraints of the EV

An individual EV is able to serve as an energy storage unit with its rapid charging and discharging characteristics. In order to dispatch the EVs under the V2B control effectively, the charging and discharging power is defined as the power output of the EV, and a negative (positive) value of the power output represents the charging (discharging) process. The operation area of an individual EV is shown in Fig. 2.

For an individual EV, the operation area (the shaded area shown in Fig. 2) is limited by the boundaries of power output and the boundaries of state of charge (SOC). Points $A, B, C, D, E$ and $F$ are used to describe the upper and lower boundaries of the operation area. 


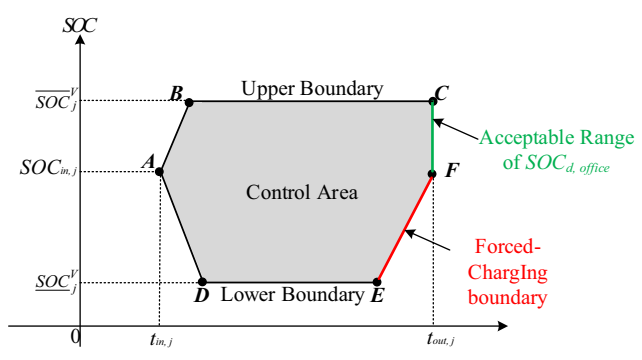

Fig. 2. The operation area of an individual EV.

The charging boundaries of ' $A-B$ ' and ' $A-D$ ' are natural boundaries constrained by the rated charging and discharging power of an individual $\mathrm{EV}$; the charging boundaries of ' $D-E$ ' and ' $B-C$ ' are natural boundaries constrained by the EV battery maintenance constraints, as shown in Eq. (4); the charging boundary of ' $E-F$ ' is a forced boundary to guarantee the minimum expected SOC $\left(S O C_{j, d, \text { office }}\right)$ at $t_{\text {out }}$; and the charging boundary of ' $C-F$ ' is a flexible boundary with acceptable range of $S O C_{j, d, \text { office, }}$ as shown in Eq. (5).

$$
\begin{aligned}
& \underline{S O C}_{j}^{V} \leq S O C_{j, t} \leq \overline{S O C}_{j}^{V} \\
& S O C_{j, t_{\text {out }}} \geq S O C_{j, d, \text { office }}
\end{aligned}
$$

Then, the real-time power output of the V2B system $\left(P_{\mathrm{V} 2 \mathrm{~B}, t}\right)$, the upper boundary $\left(P_{\mathrm{V} 2 \mathrm{~B}, t}^{\text {upper }}\right)$ and the lower boundary $\left(P_{\mathrm{V} 2 \mathrm{~B}, t}^{\text {lower }}\right)$ of the $P_{\mathrm{V} 2 \mathrm{~B}, t}$ under the $\mathrm{V} 2 \mathrm{~B}$ control are shown in Eq. (6).

$$
\left\{\begin{array}{c}
P_{\mathrm{V} 2 \mathrm{~B}, t}=\sum_{j=1}^{N_{t}} P_{j, t}^{V} \\
P_{\mathrm{V} 2 \mathrm{~B}, t}^{\text {upper }}=\sum_{j=1}^{m_{t}}\left(-P_{j, t}^{V, c}\right)+\sum_{j=1}^{n_{t}} \bar{P}_{j, t}^{V} \\
P_{\mathrm{V} 2 \mathrm{~B}, t}^{\text {lower }}=\sum_{j=1}^{m_{t}}\left(-P_{j, t}^{V, c}\right)+\sum_{j=1}^{n_{t}} \underline{P}_{j, t}^{V}
\end{array}\right.
$$

$N_{t}$ is the total number of EVs at time $t ; m_{t}$ is the number of EVs operating on the forced-charging boundary (' $E-F$ ') at time $t ; n_{t}$ is the number of the other EVs $\left(n_{t}=N_{t}-m_{t}\right)$.

\section{Formulations of the tie-line power smoothing method}

\subsection{Framework of the tie-line power smoothing method}

Due to the forecasting errors of the renewable generation, electric load demand, outdoor temperature and solar radiation, mismatches exist in both day-ahead and intrahour schedules of the tie-line power. Therefore, a twolayer tie-line power smoothing method is conducted to balance the mismatches in both short term (15 minutes) and ultra-short term (1 minute). At the master layer, the optimal dispatch program is used to generate operating schedules for the VESS (charging/discharging power of the VESS) and the V2B system (charging/discharging power of all EVs). At the client layer, the operation information of the VESS (occupied hours of the building and the indoor temperature comfort zone) as well as the upper and lower boundaries of power output of the V2B system are uploaded to the master.

\subsection{Formulations of the tie-line power smoothing method}

Considering the different response characteristics of the VESS and V2B system, different dispatch intervals are set for the VESS (short dispatch interval in 15 minutes) and the V2B system (ultra-short dispatch interval in 1 minute).

\subsubsection{Tie-line power smoothing in 15 minutes}

An optimal dispatch program is used to dispatch the VESS in 15 minutes to make the actual tie-line power schedules follow the day-ahead tie-line power schedules. Then, the tie-line power can be smoothed in 15 minutes. The optimal dispatch program can be found in our previous work 78 . The dispatch objective in 15 minutes is formulated in Eq. (7).

$$
\min \left\{\left[P_{e x, t^{\prime}}-P_{e x, t^{\prime}}^{s e t}\right]^{2}\right\}, \quad \forall t^{\prime} \in T^{\prime}
$$

where $P_{e x, t^{\prime}}^{\text {set }}$ is the day-ahead set-point of the tie-line power in time slot $t^{\prime}$, which is generated in the day-ahead dispatch stage; $P_{e x, t^{\prime}}$ is the actual tie-line power in time slot $t^{\prime}$. The constraints of the dispatch problem are the same as Eqs. (8) - (15), as shown in 8.

\subsubsection{Tie-line power smoothing in 1 minute}

The V2B system is dispatched in 1 minute to further smooth the fluctuations of the tie-line power. Firstly, call the optimal dispatch program in 1 minute to obtain the V2B control signals (i.e., the target power output of the V2B system, $P_{\mathrm{V} 2 \mathrm{~B}, t^{\prime \prime}}^{t a}$. The dispatch objective in 1 minute with the V2B control is formulated in Eq. (8). Then, the control signals are issued to all the EVs to update their power outputs.

$$
\min \left\{\left[P_{e x, t^{\prime \prime}}-P_{e x, t^{\prime \prime}}^{s e t}\right]^{2}\right\}, \quad \forall t^{\prime \prime} \in T^{\prime \prime}
$$

where $P_{e x, t^{\prime \prime}}^{s e t}$ is the day-ahead set-point of the tie-line power in time slot $t^{\prime \prime}$, which is generated in the day-ahead dispatch stage; $P_{e x, t^{\prime \prime}}$ is the actual tie-line power in time slot $t^{\prime \prime}$. The constraints consist of Eqs. (8) - (15) in Ref. 8 and Eq. (9).

$$
P_{\mathrm{V} 2 \mathrm{~B}, t^{\prime \prime}}^{\text {lower }} \leq P_{\mathrm{V} 2 \mathrm{~B}, t^{\prime \prime}}^{\text {tar }} \leq P_{\mathrm{V} 2 \mathrm{~B}, t^{\prime \prime}}^{\text {upper }}
$$

The upper boundary $\left(P_{\mathrm{V} 2 \mathrm{~B}, t^{\prime \prime}}^{\text {upper }}\right)$ and the lower boundary $\left(P_{\mathrm{V} 2 \mathrm{~B}, t^{\prime \prime}}^{\text {lower }}\right)$ of the $P_{\mathrm{V} 2 \mathrm{~B}, t^{\prime \prime}}$ are updated in every time slot after the $\mathrm{V} 2 \mathrm{~B}$ control, which is introduced in the following text. The initial boundaries (when $t^{\prime \prime}=1$,) are obtained based on the V2B model without V2B control. Based on the updated upper and lower boundary of the $P_{\mathrm{V} 2 \mathrm{~B}, t^{\prime \prime}}$, the energy management system solves the stage 2 dispatch problem in every time slot to generate the $P_{\mathrm{V} 2 \mathrm{~B}, t^{\prime \prime}}^{t a r}$ in 1 minute.

In order to realize the target power output of the V2B system $\left(P_{\mathrm{V} 2 \mathrm{~B}, t^{\prime \prime}}^{t a r}\right)$ under the V2B control, the power output of the V2B system $\left(P_{\mathrm{V} 2 \mathrm{~B}, t^{\prime \prime}}\right)$ after V2B control should

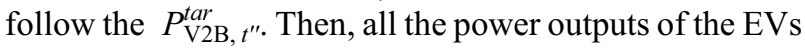
are dispatched considering their operation constraints, as discussed in Section 2.3.3. Thus, the target power output change of the V2B system is determined by Eq. (10).

$$
\Delta P_{\mathrm{V} 2 \mathrm{~B}, t^{\prime \prime}}^{t a r}=P_{\mathrm{V} 2 \mathrm{~B}, t^{\prime \prime}}^{t a r}-P_{\mathrm{V} 2 \mathrm{~B}, t^{\prime \prime}}
$$


Then, if $\Delta P_{\mathrm{V} 2 \mathrm{~B}, t^{\prime \prime}}^{\mathrm{tar}}$ is equal to zero, there is no need to adjust the power output of any. Otherwise, the following steps are conducted to determine the updated power output of each $\mathrm{EV}$ in the V2B control:

Step 1) If $\Delta P_{\mathrm{V} 2 \mathrm{~B}, t^{\prime \prime}}^{t a r}$ is higher than zero, the target power output of each EV is determined by Eq. (11). And $m_{1, t}$ is defined as the number of EVs of the V2B system which have not reached their upper limits of power output,

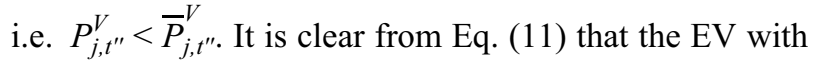
higher SOC level will contribute more discharging power to the positive $\Delta P_{\mathrm{V} 2 \mathrm{~B}, t^{\prime \prime}}^{t a r}$ value.

$$
P_{j, t^{\prime \prime}}^{V, t a r}=P_{j, t^{\prime \prime}}^{V}+\Delta P_{\mathrm{V} 2 \mathrm{~B}, t^{\prime \prime}}^{\text {tar }} \times \frac{S O C_{j, t^{\prime \prime}}^{V}-\underline{S O C_{j}^{V}}}{\sum_{j=1}^{m_{1, t}}\left(S O C_{j, t^{\prime \prime}}^{V}-\underline{S O C}_{j}^{V}\right)}
$$

Step 2) If $\Delta P_{\mathrm{V} 2 \mathrm{~B}, t^{\prime \prime}}^{t a r}$ is lower than zero, the target power output of each EV is determined by Eq. (12). And $m_{2, t}$ is defined as the number of EVs in the V2B system which have not reached their lower limits of power output,

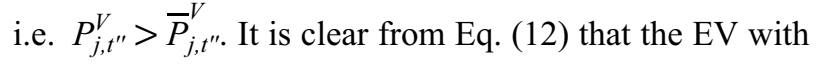
lower SOC level will contribute more charging power to the negative $\Delta P_{\mathrm{V} 2 \mathrm{~B}, t^{\prime \prime}}^{t a r}$ value.

$$
P_{j, t^{\prime \prime}}^{V, t a r}=P_{j, t^{\prime \prime}}^{V}+\Delta P_{\mathrm{V} 2 \mathrm{~B}, t^{\prime \prime}}^{t a r} \times \frac{\overline{S O C}_{j}^{V}-S O C_{j, t^{\prime \prime}}^{V}}{\sum_{j=1}^{m_{2, t}}\left(\overline{S O C}_{j}^{V}-S O C_{j, t^{\prime \prime}}^{V}\right)}
$$

each EV, $m_{3, t}\left(m_{4, t}\right)$ is defined as the number of EVs which have violated their upper (lower) power output limits. Thus, the new revised $\Delta P_{\mathrm{V} 2 \mathrm{~B}, t^{\prime \prime}}^{\text {tar }}$ is determined by Eq. (13). If $\Delta P_{\mathrm{V} 2 \mathrm{~B}, t^{\prime \prime}}^{t a r}$ is equal to zero, the power output of $\mathrm{EV} j$ after $\mathrm{V} 2 \mathrm{~B}$ control is updated with $P_{j, t^{\prime \prime}}^{V, t a r}$. If $\Delta P_{\mathrm{V} 2 \mathrm{~B}, t^{\prime \prime}}^{t a r}$ is not equal to zero, Step 1) $\sim$ Step 3) will be implemented again until the updated $\Delta P_{\mathrm{V} 2 \mathrm{~B}, t^{\prime \prime}}^{t a r}$ is equal to zero.

$$
\left\{\begin{array}{l}
\Delta P_{\mathrm{V} 2 \mathrm{~B}, t^{\prime \prime}}^{\text {tar }}=\sum_{j=1}^{m_{3, t}}\left(P_{j, t^{\prime \prime}}^{V, \text { tar }}-\bar{P}_{j, t^{\prime \prime}}^{V}\right), m_{3, t}>0 \& m_{4, t}=0 \\
\Delta P_{\mathrm{V} 2 \mathrm{~B}, t^{\prime \prime}}^{\text {tar }}=\sum_{j=1}^{m_{4, t}}\left(P_{j, t^{\prime \prime}}^{V, t a r}-\underline{P}_{j, t^{\prime \prime}}^{V}\right), m_{3, t}=0 \& m_{4, t}>0
\end{array}\right.
$$

\section{Case studies}

\subsection{Case description}

A building Microgrid shown in Fig. 1 is used to verify the effectiveness of the developed tie-line power smoothing method. The building is represented by a parallelepiped with a squared floor. The thermal parameters of the building are given in Tab. 17 . The occupied hours are set to be from $8: 00 \mathrm{a} . \mathrm{m}$. to $20: 00$ p.m 15 . In this study, the indoor temperature set-point range is set to be from $19^{\circ} \mathrm{C}$ to $26^{\circ} \mathrm{C}$ with VESS being dispatched.

Step 3) Based on the obtained target power output of

Table 1. Building parameters.

\begin{tabular}{ccccccc}
\hline \hline$U_{\text {wall }}\left[\mathrm{W} /\left(\mathrm{m}^{2} \cdot \mathrm{K}\right)\right]$ & $F_{\text {wall }}\left(\mathrm{m}^{2}\right)$ & $U_{\text {win }}\left[\mathrm{W} /\left(\mathrm{m}^{2} \cdot \mathrm{K}\right)\right]$ & $\begin{array}{c}\text { Window to } \\
\text { wall ratio }(\%)\end{array}$ & $\begin{array}{c}\text { Long } \\
\text { side }(\mathrm{m})\end{array}$ & $\begin{array}{c}\text { Short } \\
\text { side }(\mathrm{m})\end{array}$ & Height $(\mathrm{m})$ \\
\hline 0.908 & 2400 & 2.750 & 75 & 40 & 20 & 30 \\
\hline \hline
\end{tabular}

The measured actual outdoor temperature in a summer day are shown in Fig. 3 7. The forecasted and actual solar radiation on horizontal surface 16 are shown in Fig. 4 . The rated power of photovoltaic system is $100 \mathrm{~kW}$. The forecasted and actual electric loads and internal heat gains

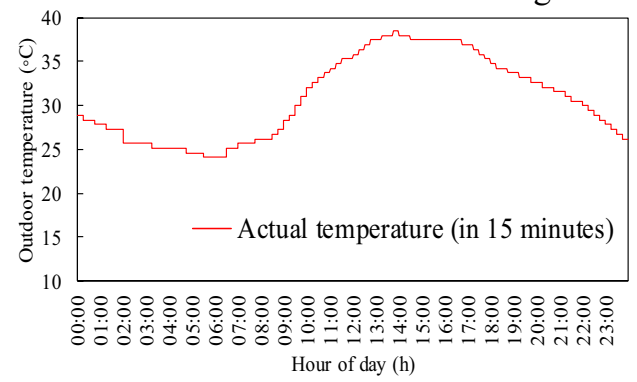

Fig. 3. Solar radiation on horizontal surface. of the building in a typical summer day are shown in Fig. 5 and Fig. 6 respectively. The number of EVs is assumed be 10 in this case study. The rated charging and discharging power of an individual EV is $3.3 \mathrm{~kW} 17$.

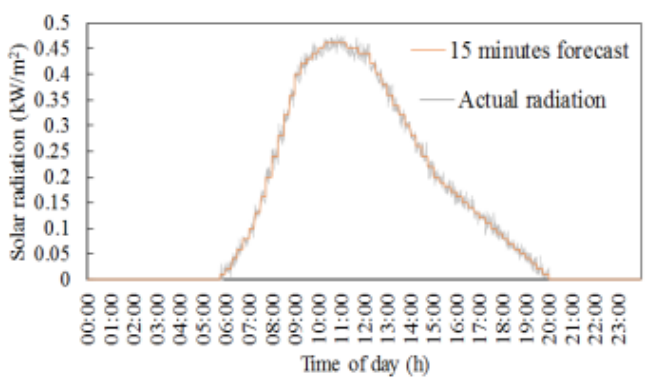

Fig. 4. Solar radiation on horizontal surface. 


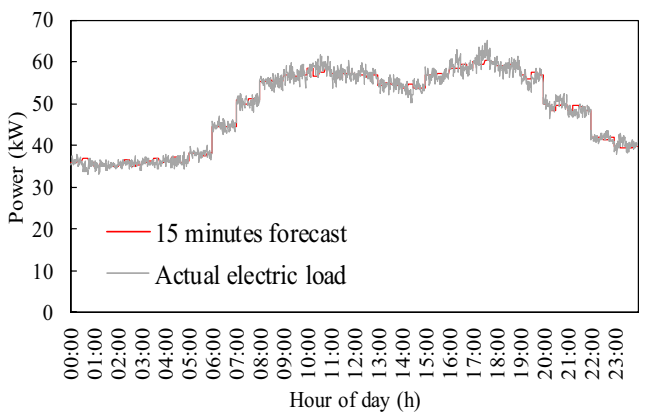

Fig. 5. Electric load of the building Microgrid.

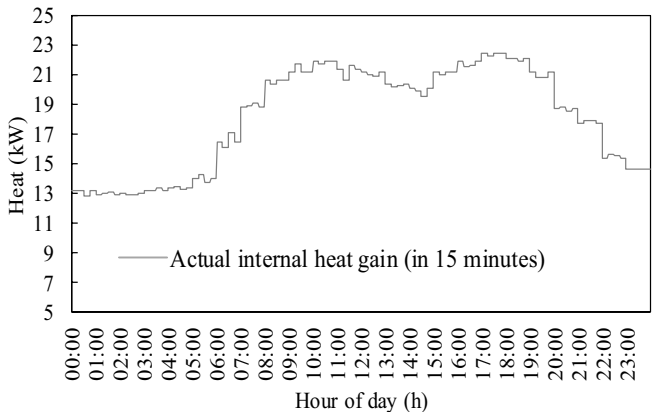

Fig. 6. Internal heat gain of the building.

The day-ahead set-point of the tie-line power is shown by the black solid line in Fig. 7, which is generated in the day-ahead dispatch stage. The comparative dispatch results in Scenario I (represented by the orange solid line in Fig. 7) and Scenario II (represented by the grey solid line in Fig. 7) suggest that the fluctuations of the tie-line power can be reduced to some extend using the short-term dispatch of VESS in 15 minutes. Since all the mismatches between the energy demand and supply are balanced by electric power from the external grid under the DA-P method in Scenario I, all the forecasting errors are mainly reflected in the electric power fluctuations at the tie-line, as shown in Fig. 7. short-term dispatch of the VESS at the actual operation stage. The VESS is dispatched to smooth the fluctuations of the tie-line power.

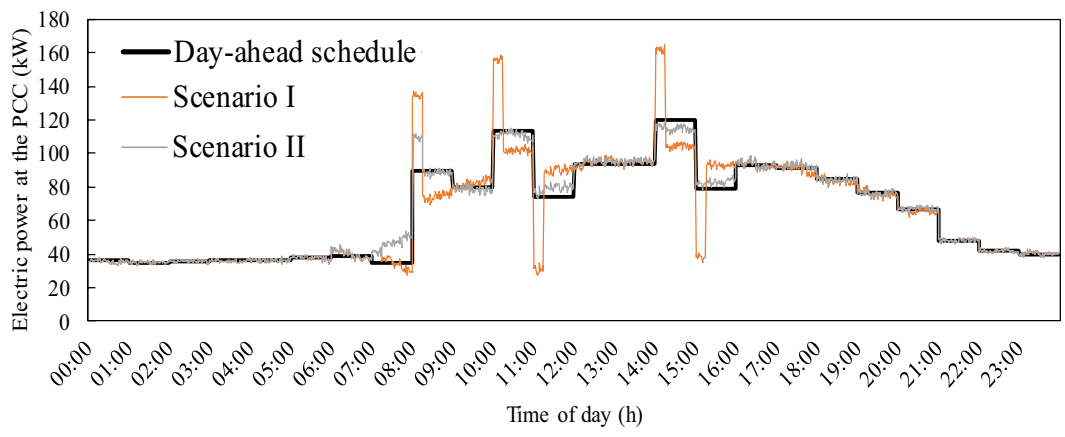

Fig. 7. Tie-line power of the building Microgrid.

The dispatch results of the cooling demand with indoor temperature set-point of the building Microgrid in Scenario II with VESS dispatched is depicted in Fig. 8. The indoor temperature set-point schedules fluctuate within the indoor temperature set-point comfort range (19

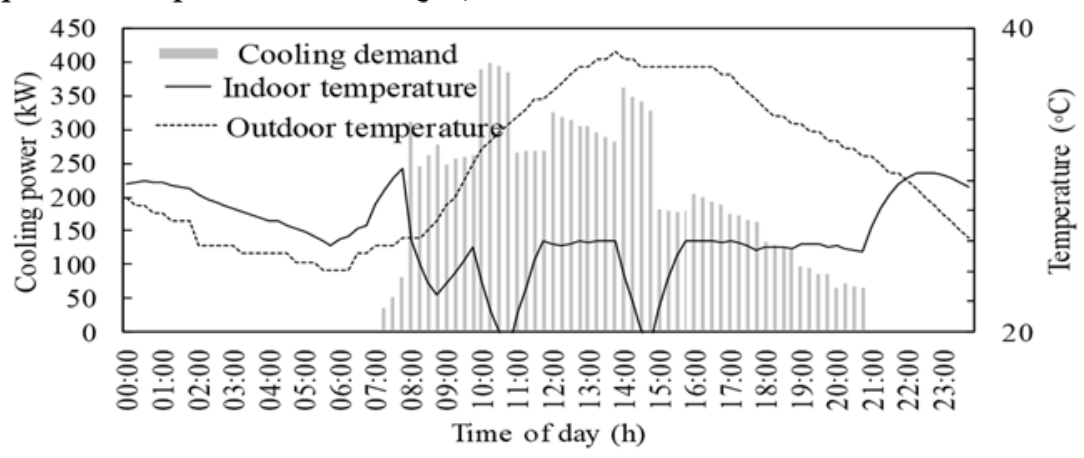

Fig. 8. The cooling demand and indoor temperature of the building with VESS dispatched.
${ }^{\circ} \mathrm{C} \sim 26{ }^{\circ} \mathrm{C}$ ) during the occupied hours in Scenario II. The cooling demand of the building is also dispatched under Scenario II to dispatch the VESS for smoothing the fluctuations of the tie-line power. 


\subsubsection{V2B dispatch results}

Scenario III is further developed to verify the effectiveness of the method with ultra-short term dispatch (in 1 minute) of the V2B system.
A comparison of the dispatch results in Scenario III (red solid line in Fig. 9), Scenario I (orange solid line in Fig. 9) and Scenario II (grey solid line in Fig. 9) suggests that the electric power fluctuations at the PCC are well smoothed by the V2B system.

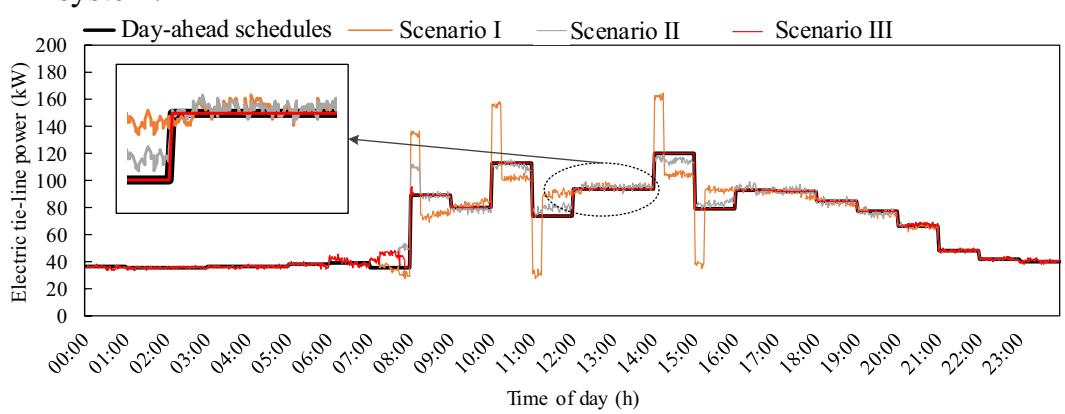

Fig. 9. Tie-line power of the building Microgrid in the comparative scenarios.

The ultra-short term dispatch results of the V2B system in 1 minute is shown in Fig. 10. The optimal dispatch program (as shown in Eq. (8)) is implemented to generate the target power output of the V2B system (represented by the grey solid line in Fig. 10) based on the updated upper boundary (represented by the black solid line in Fig. 10) and the lower boundary (represented by the green solid line in Fig. 10) of the real-time power output of the V2B system. And the target power output of

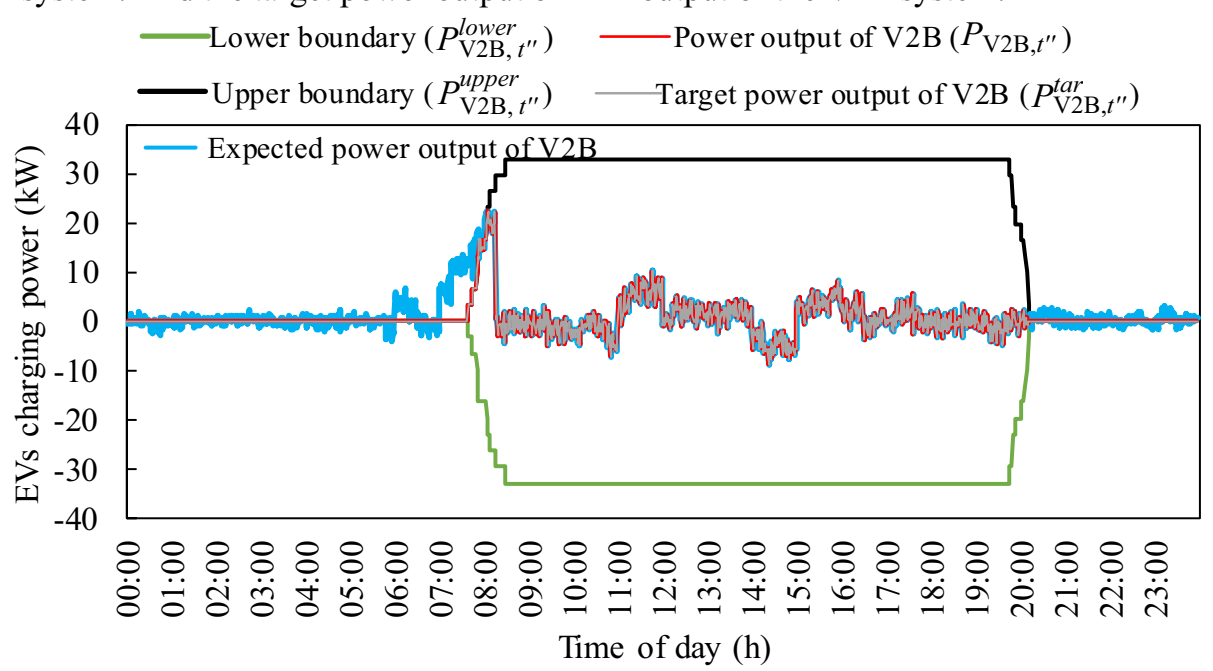

Fig. 10. The ultra-short term dispatch results of the V2B system in 1 minute.

\section{Conclusion}

This paper proposes a two-layer tie-line power smoothing method for a building microgrid by scheduling thermal mass of the building and EVs. To incorporate the different response characteristics and the forecasting errors of the input data at different time scales, the VESS and the V2B system are coordinated at two different time scales. At the master level, the VESS is dispatched within a short-term in 15 minutes. A V2B control strategy is further developed to dispatch the power output of the V2B system within an ultra-short term in 1 minute. At the client level, the operation information of the VESS as well as the upper and lower boundaries of the V2B system are the V2B system is broadcasted to the EVs in every 1 minute. Then, all the EVs update their power outputs according to the target power output of the V2B system and the V2B control strategy. The simulation results shown in Fig. 10 reveal that the V2B system is dispatched within the V2B power output limits. And the real-time power output of the V2B system (represented by the red solid line in Fig. 10) can track the real-time target power output of the V2B system.

Time of day $(\mathrm{h})$

generated and uploaded to the master level. Numerical studies show that the proposed method can smooth the fluctuations of the tie-line power of the building Microgrid.

\section{Acknowledgements}

This work was financially funded by the Science and Technology Project of State Grid Corporation of China (SGTJJY00GHJS1900040).

\section{References}

1. J. Keirstead, M. Jennings and A. Sivakumar, "A 
review of urban energy system models: Approaches, challenges and opportunities," Renew. Sust. Energ. Rev., vol. 16, no. 6, pp. 3847-3866, 2012.

2. D. H. W. Li, S. K. H. Chow, E. W. M. Lee, "An analysis of a medium size grid-connected building integrated photovoltaic (BIPV) system using measured data," Energy Build., vol. 60, no. 5, pp. 383-387, 2013.

3. X. Guan, Z. Xu and Q. Jia, "Energy-Efficient Buildings Facilitated by Microgrid," IEEE Trans. Smart Grid, vol. 1, no. 3, pp. 243-252, 2010.

4. D. Zhang, S. Evangelisti, P. Lettieri, et al, "Economic and environmental scheduling of smart homes with microgrid: DER operation and electrical tasks," Energy Convers. Manage., vol. 110, no. Supp14, pp. 113-124, 2016.

5. Y. Zhao, Y. Lu, C. Yan, et al, "MPC-Based Optimal Scheduling of Grid-Connected Low Energy Buildings with Thermal Energy Storages," Energy Build., vol. 86, no. 12, pp. 415-426, 2014.

6. JA. Duffie and WA. Beckman, "Solar engineering of thermal process," New York: Wiley, 1991.

7. X. Jin, Y. Mu, H. Jia, et al, "Dynamic economic dispatch of a hybrid energy microgrid considering building based virtual energy storage system," Appl. Energy, vol. 194, no. 1, pp. 386-398, 2017.

8. X. Jin, X. Wang, Y. Mu, et al, "Optimal scheduling approach for a combined cooling, heating and power building microgrid considering virtual storage system," Power and Energy Society General Meeting, IEEE, pp. 1-5, 2016.

9. Y. $\mathrm{Mu}, \mathrm{J} . \mathrm{Wu}, \mathrm{J}$. Ekanayake, et al, "Primary Frequency Response From Electric Vehicles in the Great Britain Power System," IEEE Trans. Smart Grid, vol. 4, no. 2, pp. 1142-1150, 2013.

10. EU Merge Project. Deliverable 2.1: "Modelling Electric Storage devices for electric vehicles, 2010," [Online]. Available: http://www.ev-merge.eu/ images stories/uploads/MERGE_WP2_D2.1.pdf.

11. M. Wang, Y. Mu, H. Jia, et al, "Active power regulation for large-scale wind farms through an efficient power plant model of electric vehicles," Appl. Energy, vol. 185, no. 1, pp. 1673-1683, 2017.

12. X. Dong, Y. Mu, H. Jia, et al, "Planning of Fast EV Charging Stations on a Round Freeway," IEEE Trans. Sustain. Energy, vol. 7, no. 4, pp. 1452-1461, 2016.

13. Z. Wang, L. Wang, A. I. Dounis, et al, "Integration of plug-in hybrid electric vehicles into energy and comfort management for smart building," Energy Build., vol. 47, no. 47, pp. 260-266, 2012.

14. M. Ardeshir, M. Abdolazim, K. Elham, et al, "Occupants' operation of lighting and shading systems in buildings," J. Build. Perform. Simu., vol. 1, no. 1, pp. 57-65, 2008.

15. D. Zhang, N. Shah and L. G. Papageorgiou, "Efficient energy consumption and operation management in a smart building with microgrid," Energy Convers. Manage., vol. 74, no. 74, pp. 209-
222, 2013.

16. Kaşka Önder and R. Yumrutaş, "Experimental investigation for total equivalent temperature difference (TETD) values of building walls and flat roofs," Energy Convers. Manage., vol. 50, no. 11, pp. 2818-2825, 2009.

17. J. Van Roy, N. Leemput, F. Geth, et al, "Electric Vehicle Charging in a building Microgrid with Distributed Energy Resources," IEEE Trans. Sustain. Energy, vol. 5, no. 4, pp. 1-8, 2014. 